\title{
DIFFERENCE IN EFFECTIVE DIFFUSIVITY UNDER REACTIVE AND NON-REACTIVE DIFFUSION CONDITIONS*
}

\author{
NORIAKI WAKAO \\ Yokohama National University, Yokohama
}

\begin{abstract}
On the basis of a pore model, a difference in average pore radius under reactive and non-reactive diffusion conditions is discussed. This model suggests a difference in intraparticle effective diffusivity value (pore volume diffusivity with no surface diffusion contribution) between these two systems. Experimental pore tortuosity measurements are also reported for commercial catalyst pellets.
\end{abstract}

Calculation of the effective diffusivity in porous catalyst is important in evaluating the contribution of intraparticle diffusion to the total reaction rate. Many studies have been reported of experimental measurement and estimation of the effective diffusivity, as reviewed excellently by Satterfield and Sherwood ${ }^{73}$ and by Petersen ${ }^{4}$. However, less attention has been focused on whether the effective diffusivity value for the non-reactive diffusion system is identical with that for the reacting system. The object of this paper is to explore any difference in average pore radius and that in effective diffusivity value between these two systems.

\section{Average Pore Radius and Effective Diffusivity-Pore Model}

Let us consider a porous medium made up of an array of straight pores different in diameter (denoted as pellet I). On the other hand, suppose a bundle of such uniform straight capillary tubes (radius $\bar{a}^{R}$ ) such that the catalytic reaction rate equals that in pellet $I^{9)}$. Hence, the radius $\vec{a}^{R}$ is defined as the average pore radius of pellet I under the reacting condition.

If the catalytic reaction is first order, the reaction rate $r(a)$ in a single capillary tube (radius $=a$, length $=L$ ) is

$$
r(a)=2 \pi a L k_{s} C_{i o} E_{f}(a)
$$

Let the function $n(a) d a$ denote the number of pores per unit mass of pellet $\mathrm{I}$, with a radius between $a$ and $a+d a$. The total reaction rate of pellet I (cross sectional area $=A_{s}$, length $=L$, density $\left.=\rho_{P}\right)$ is

$$
A_{S} L \rho_{P} \int_{0}^{\infty} r(a) n(a) d a=2 A_{s} L \rho_{P} k_{s} C_{i 0} \int_{0}^{V_{t}} \frac{E_{f}(a)}{a} d V
$$

where $d V=\pi a^{2} \operatorname{Ln}(a) d a$.

The reaction rate in the bundle of uniform capillary tubes (radius $=\bar{a}^{R}$, volume void fraction of the tubes $\left.=f^{R}\right)$ is

$$
2 A_{s} L f^{R} k_{S} C_{i o} E_{f}\left(\bar{a}^{R}\right) / \bar{a}^{R} .
$$

In earlier papers ${ }^{9,12)} f^{R}$ was called as effective area void fraction, but it should read the effective volume void fraction in consideration of applying to tortuous pores

* Received on July 15, 1969

Presented at The IV International Congress on Catalysis, Novosibirsk Symposium, July 1968 in actual catalyst pellet.

Equating Eq. (2) to Eq. (3), we obtain

$$
\int_{0}^{V_{t}} \frac{E_{f}(a)}{a} d V=\frac{E_{f}\left(\bar{a}^{R}\right)}{\bar{a}^{R}} \frac{f^{R}}{\rho_{P}} \text {. }
$$

The effectiveness factor $E_{f}$ is a function of the Thiele modulus $\phi \equiv(L / 2) \sqrt{2 k_{s} / a D_{i}^{R}(a)}$.

Hence, Let us consider two pellets, identical except for the height $L$. There is, of course, no difference in diffusivity value between these two pellets. As a result, Eq. (4) yields the following two relations, from which the two quantities $\bar{a}^{R}$ and $f^{R}$ are determined.

When $L$ is small, $E_{f}$ tends to unity, and then Eq. (4) reduces to

$$
\int_{0}^{V_{t}} \frac{d V}{a}=\frac{f^{R}}{\bar{a}^{R}} \frac{1}{\rho_{P}} .
$$

When $L$ is sufficiently large, $E_{f}$ becomes inversely proportional to $\phi ; E_{f}(a) \propto \sqrt{a D_{i}{ }^{R}(a)}$, and then Eq. (4) becomes

$$
\int_{0}^{V_{\imath}} \sqrt{\frac{D_{i}^{R}(a)}{a}} d V=\sqrt{\frac{D_{i}^{R}\left(\bar{a}^{R}\right)}{\bar{a}^{R}}} \frac{f^{R}}{\rho_{P}} .
$$

The effective diffusivity for the reacting system, in accordance with the model, is expressed as

$$
D_{e i}{ }^{R}=f^{R} \cdot D_{i}{ }^{R}\left(\bar{a}^{R}\right) \text {. }
$$

Wheeler ${ }^{11}$ has proposed the following equation for calculation of the average pore radius $\bar{a}$.

$$
a=2 V_{t} / S_{B E T} \text {. }
$$

It is easily shown that in the Knudsen diffusion region Eqs. (5) and (6) reduce to Eq. (8): When Knudsen diffusion is dominant in the pellet, $D_{i}{ }^{n}(a)=D_{K i} \propto a$, and then Eq. (6) becomes

$$
\begin{gathered}
V_{t}=f^{R} / \rho_{P} \\
\text { or } \quad \varepsilon=V_{t} \rho_{P}=f^{R} .
\end{gathered}
$$

Letting $S$ denote the surface area function per unit mass of the pellet: $d S=2 d V / a, \mathrm{Eq}$. (5) reduces to

$$
S_{t} / 2=f^{R} /\left(\tilde{a}^{R} \rho_{P}\right) \text {. }
$$

If $S_{t}\left(=\int_{0}^{s_{t}} d S=2 \int_{0}^{V_{t}} d V / a\right)=S_{B E T}$ (As shown in Table 2 , this is almost true for actual catalyst pellets having a considerable amount of micro pores), combination of Eqs. (9) and (10) yields Eq. (8).

Suppose that a pore size distribution in pellet $I$ is such as the one illustrated in Fig. 1, and that the following hypothetical catalytic reaction is carried out in the inert 
gas, nitrogen.

$\mathrm{H}_{2}+\mathrm{A} \rightarrow \mathrm{B}$ at $21^{\circ} \mathrm{C}$ and $1 \mathrm{~atm}$.

Furthermore, assuming the following average mole fractions in the pellet

$y_{\mathrm{H}_{2}}, y_{\mathrm{A}}, y_{\mathrm{B}} \ll y_{\mathrm{N}_{2}} \fallingdotseq 1$

the reactive diffusivity of hydrogen in a capillary tube of radius $a$ is simplified as (refer to Eq. (A 1) in Table 1)

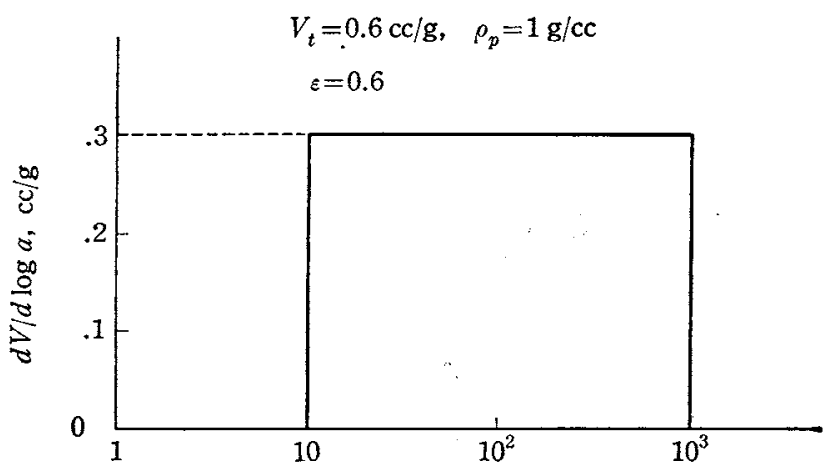

PORE RADIUS $a, \AA$

Fig. I Hypothetical pore size distribution

$$
\begin{aligned}
D_{\mathrm{H}_{2}}{ }^{R}(a) & =\frac{1}{\frac{3}{2 \bar{v}_{\mathrm{H}_{2}} a}+\frac{1}{D_{\mathrm{H}_{2}-\mathrm{N}_{2}}}} \\
& =\frac{1}{\frac{1}{0.00117 \times a[\AA]}+\frac{1}{0.640}}\left[\mathrm{~cm}^{2} / \mathrm{sec}\right]
\end{aligned}
$$

Substituting Eq. (12) into Eq. (6), and solving with Eq. (5), we obtain (refer to Fig. 2) $\bar{a}^{R}=42 \AA$ and $f^{R}$ $=0.54$. Thus, Eq. (7) gives $D_{e \mathrm{H}_{a}}{ }^{R}=0.0247 \mathrm{~cm}^{2} / \mathrm{sec}$. Note that Wheeler's Eq. (8) gives

$$
\bar{a}=\frac{2 V_{t}}{S_{B E T}}=\frac{V_{t}}{\int_{0}^{V_{t}} \frac{d V}{a}}=46.5 \AA
$$

In a non-reactive diffusion system, the effective diffusivity within pellet $I$ is ${ }^{9)}$

$$
D_{e i}{ }^{D}=L \rho_{P} \int_{0}^{\infty} D_{i}{ }^{D}(a) \pi a^{2} n(a) d a=\rho_{P} \int_{0}^{V_{t}} D_{i}{ }^{D}(a) d V
$$

Hence, again we assume a bundle of uniform straight capillary tubes through which the diffusion flux is equal to that in pellet I. The effective diffusivity in such uniform capillary tubes is expressed as

$$
D_{e i}{ }^{D}=f^{D} \cdot D_{i}{ }^{D}\left(\bar{a}^{D}\right)
$$

Hence, $\bar{a}^{D}$ is denoted the average pore radius for the non-reactive diffusion system.

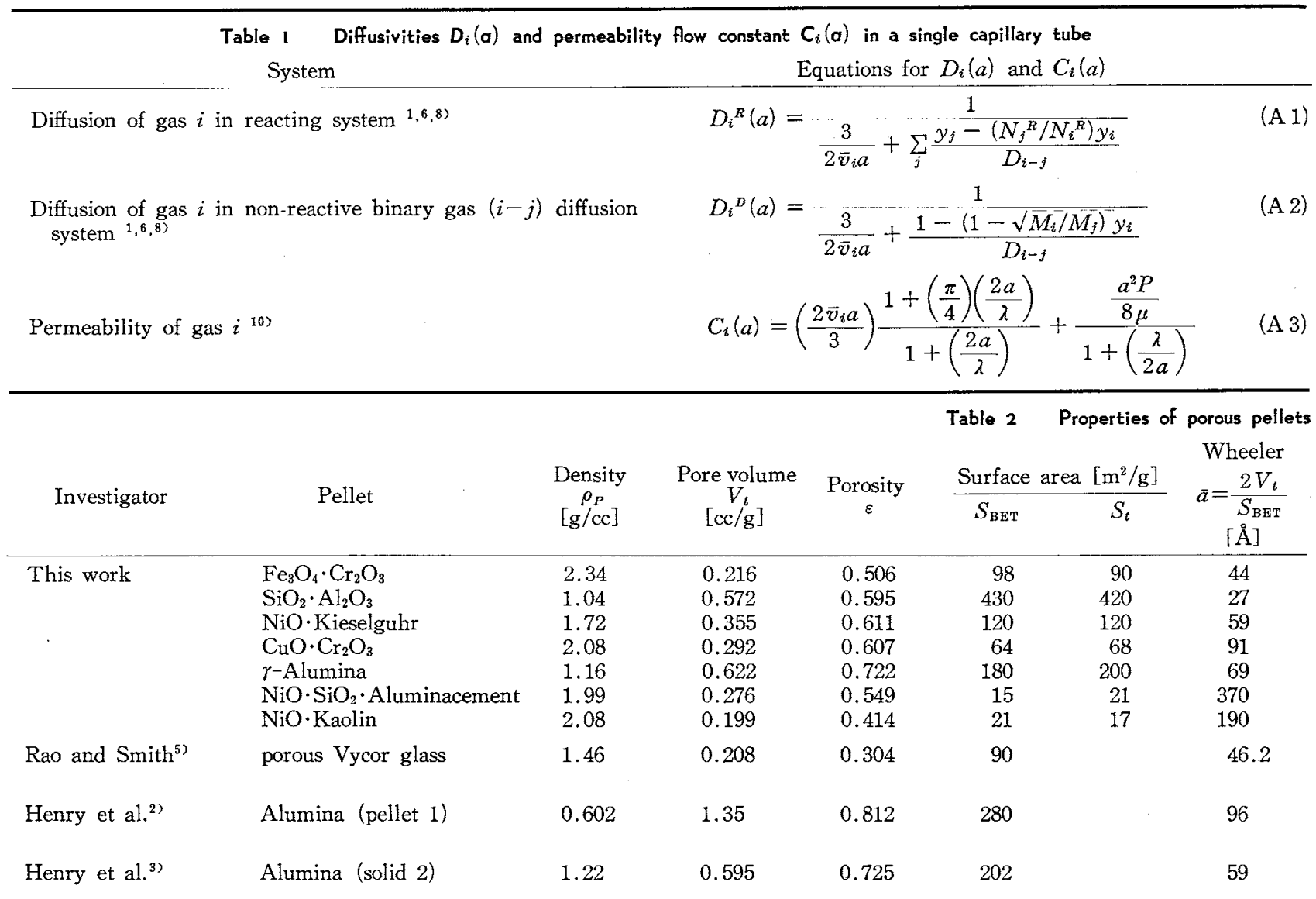

(a) calculated value, assuming $S_{t}=S_{\mathrm{BET}}$.

(b) calculated from Eq. (14), assuming $f^{D}=\varepsilon$.

(c) calculated with an aid 
Suppose that pellet $\mathrm{I}$ is tested for the diffusion experiment in a binary $\mathrm{H}_{2}-\mathrm{N}_{2}$ system, using, for example, a Wicke-Kallenbach type apparatus. If the average mole fractions in the pellets are $y_{\mathrm{H}_{2}}=y_{\mathrm{N}_{2}}=0.5$, the diffusivity of hydrogen in a capillary tube of radius $a$ becomes (refer to Eq. (A 2) in Table 1) at $21^{\circ} \mathrm{C}$

$$
D_{\mathrm{H}_{2}}{ }^{D}(a)=\frac{1}{\frac{1}{0.00117 \times a[\AA]}+\frac{0.634}{0.640}}\left[\mathrm{~cm}^{2} / \mathrm{sec}\right]
$$

Substituting Eq. (15) into Eq. (13), and using the pore volume function illustrated in Fig. 1, the effective diffusivity is evaluated as $D_{e \mathrm{H}_{2}}{ }^{D}=0.0997 \mathrm{~cm}^{2} / \mathrm{sec}$, which is about four times the $D_{e \mathrm{H}_{3}}{ }^{R}$ value.

Thus, with this result a relationship $\bar{a}^{D}-f^{D}$ is obtained from Eq. (14), and is indicated in Fig. 2. The $\bar{a}^{D}$ value cannot be evaluated, unless $f^{D}$ value is assumed. However, it should be pointed out, in any event, that the $\bar{a}^{D}$ value must be much larger than the $\bar{a}^{R}$ value.
Pore Tortuosity and Comparison of Average Pore RadiiCatalyst Pellets

In actual catalyst pellets, pores are tortuous and in random orientations. Hence, introducing the concept of the pore tortuosity factor $^{5)}, k$, the effective diffusivities in the catalyst pellets are assumed to be expressed as

$$
\begin{aligned}
D_{c i}{ }^{R} & =\frac{f^{R}}{k^{2}} D_{i}{ }^{R}\left(\bar{a}^{R}\right), \\
D_{e i}{ }^{D} & =\frac{f^{D}}{k^{2}} D_{i}{ }^{D}\left(\bar{a}^{D}\right) \\
& =\frac{\rho_{P}}{k^{2}} \int_{0}^{V} D_{i}^{D}(a) d V
\end{aligned}
$$

With the experimentally measured $D_{e^{-}}{ }^{D}$ data for commercial catalyst pellets (for pore size distributions see Fig. 3), the tortuosity factors for these pellets are evaluated from Eq. $\left(17^{\prime}\right)$, and illustrated in Table 2. Since Rao and Smith ${ }^{5)}$ on porous Vycor glass and Henry et
Fig. 2 Relationship between $\vec{a}_{R}$ and $f^{R}$, and that $\bar{a}^{D}$ and $f^{D}$

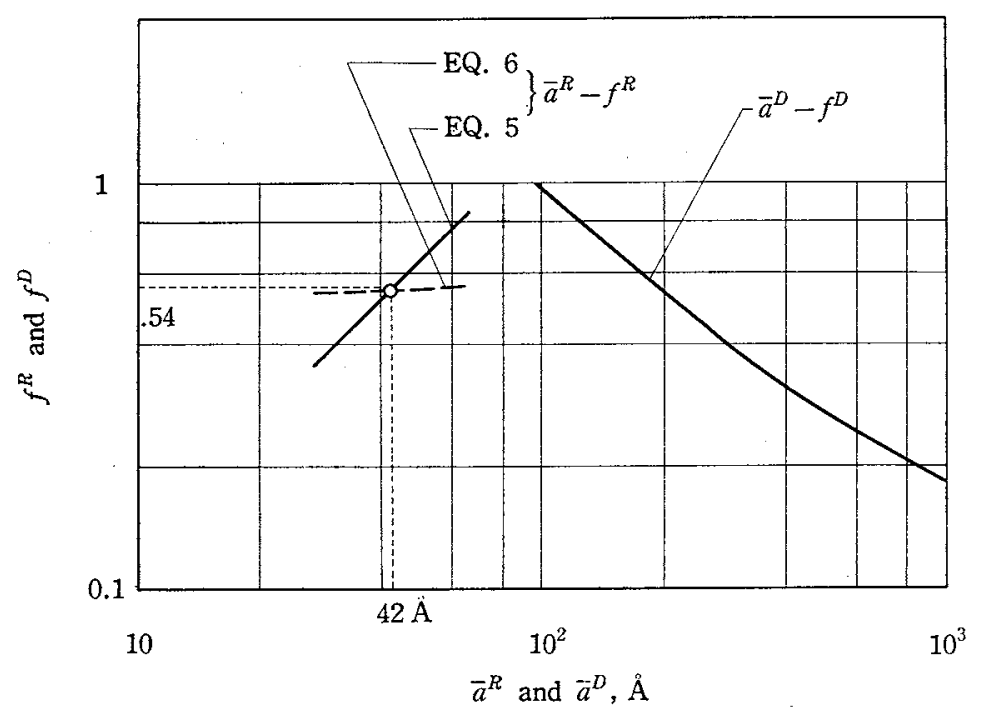

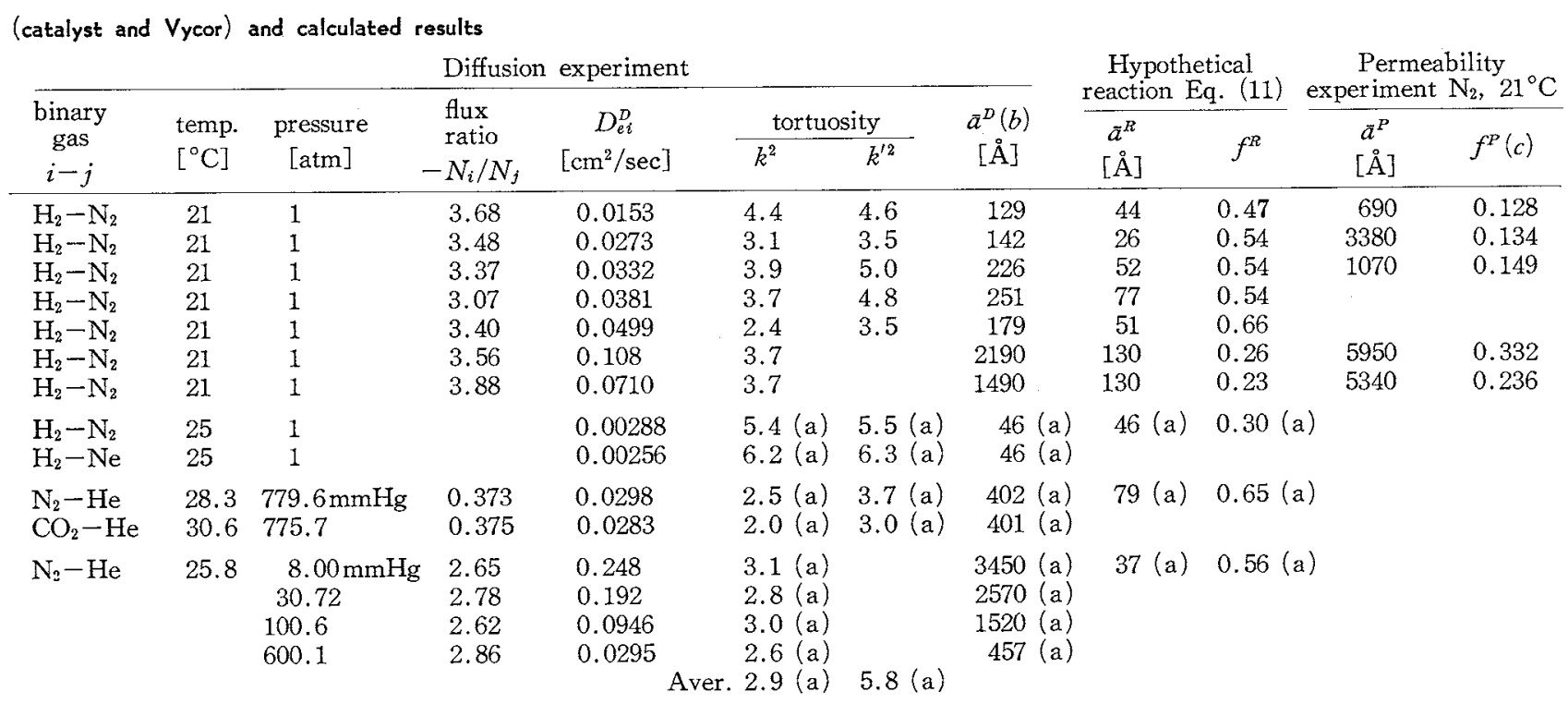

of $k^{2}$ value determined from diffusion experiment. 


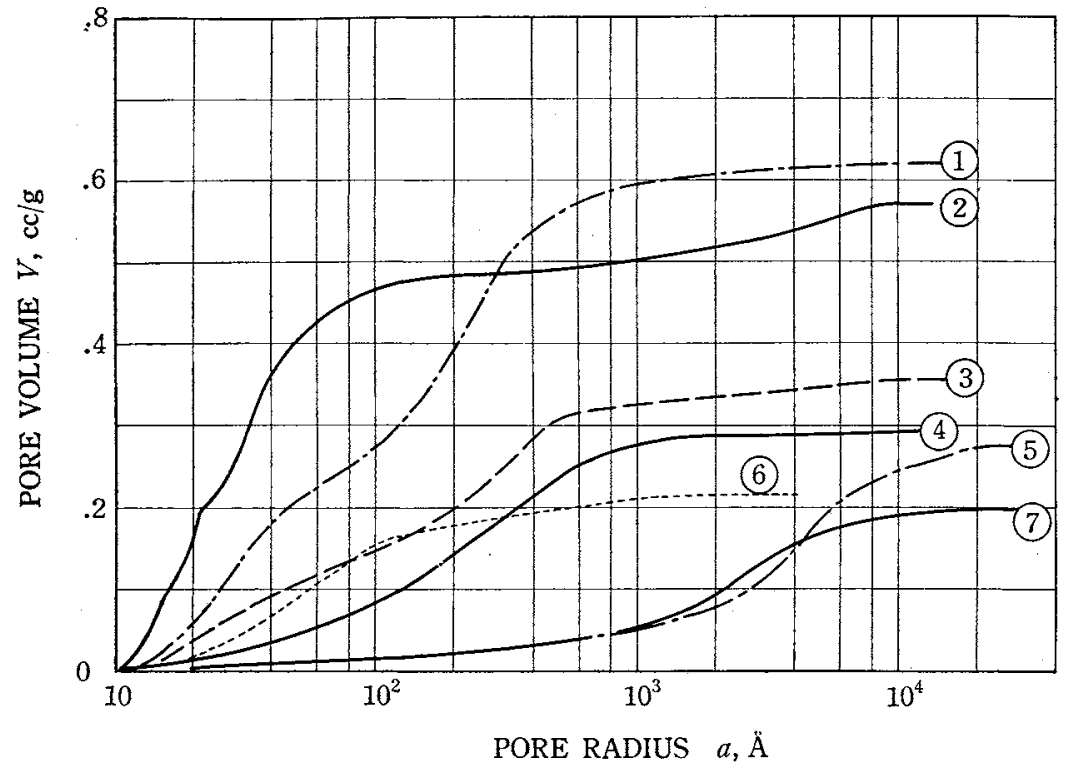

(1) $\gamma$-Alumina

(2) $\mathrm{SiO}_{2} \cdot \mathrm{Al}_{2} \mathrm{O}_{3}$

(3) $\mathrm{NiO} \cdot \mathrm{Kieselguhr}$

(4) $\mathrm{CuO} \cdot \mathrm{Cr}_{2} \mathrm{O}_{3}$

(5) $\mathrm{NiO} \cdot \mathrm{SiO}_{2} \cdot$ Aluminacement

(6) $\mathrm{Fe}_{3} \mathrm{O}_{4} \cdot \mathrm{Cr}_{2} \mathrm{O}_{3}$

(7) $\mathrm{NiO} \cdot \mathrm{Kaolin}$

Fig. 3 Cumulative pore size distribution curves for catalyst pellets $\mathrm{al}^{2,37}$ on alumina pellets have shown both the diffusion experimental data and the pore size distribution curves, the $k^{2}$ values for their pellets are also calculated and compared.

In the reacting system, the values of $\bar{a}^{R}$ and $f^{R}$ may be determined from Eqs. (5) and (6). With these values and the $k^{2}$ values, the effective diffusivities are evaluated from Eq. (16).

As shown already, $\bar{a}^{R}=\bar{a}$ and $f^{R}=\varepsilon$ in the Knudsen diffusion region. Thus, except for pellets $(\bar{a}>100 \AA)$ such as alumina-cement and kaolin carriers having almost entirely macro pores, the reactive diffusivity for the pellets having a considerable amount of micro pores may be approximately expressed as

$$
\begin{gathered}
D_{e i}{ }^{R}=\frac{\varepsilon}{k^{\prime 2}} D_{i}{ }^{R}\left(\bar{a}=2 V_{t} / S_{B E T}\right), \\
\quad \text { for pellets of } \bar{a}<100 \AA
\end{gathered}
$$

The $k^{\prime 2}$ values ( $k^{\prime}$ : modified tortuosity factor) thus defined are shown in Table 2.

On the other hand, let us consider an average pore radius under gas permeability conditions. If the permeability flux through a porous medium can be expressed as that through a bundle of uniform capillary tubes (of tortuosity factor $k$, radius $\bar{a}^{p}$ and volume void fraction $\left.f^{P}\right)$, it follows that

$$
Q_{i}=-\frac{f^{P}}{k^{2}} \frac{C_{i}\left(\tilde{a}^{P}\right)}{R_{g} T} \frac{d P}{d x}
$$

Substituting Eq. (A 3) (Table 1) into Eq. (19) and integrating between $P^{\prime}\left(=P^{\prime \prime}+\Delta P\right)$ at $x=0$ and $P^{\prime \prime}$ at $x=L$, we obtain

$$
\begin{aligned}
& \frac{Q_{i} R_{g} T L}{\Delta P}=\alpha+\beta \frac{\Delta P}{P_{0}} \\
& \alpha=\frac{f^{P}}{k^{2}} \frac{2 \bar{v}_{i} \bar{a}^{P}}{3}\left[0.7118+0.0736\left(\frac{2 \bar{a}^{P}}{\lambda_{0}}\right)\left(\frac{P^{\prime \prime}}{P_{0}}\right)\right] \\
& \beta=\frac{f^{p}}{k^{2}} \frac{2 \bar{v}_{i} \bar{a}^{P}}{3}(0.0368)\left(\frac{2 \bar{a}^{P}}{\lambda_{0}}\right)
\end{aligned}
$$

where $P_{0}$ is the atmospheric pressure and $\lambda_{0}$ is the mean free path at $P_{0}$. When the flow rate data are plotted as $Q_{i} / \Delta P$ against $\Delta P / P_{0}$, a straight line will be drawn. Thus, $f^{P} / k^{2}$ and $\bar{a}^{P}$ values are evaluated from the slope and intercept of the straight line. As illustrated in Table 2, the average pore radius is found to be entirely dependent on the system; $\bar{a}^{R}<\bar{a}^{D}<\bar{a}^{P}$, for the catalyst pellets having broad pore size distributions. However, for pellets (such as porous Vycor glass) having relatively uniform pores, it is obvious that the radius of the uniform pores $=\bar{a}^{R}=\bar{a}^{D}=\bar{a}^{P}$ and that $\varepsilon=f^{R}=f^{D}=f^{P}$.

The application of the present investigation in obtaining the effective diffusivities under reactive and nonreactive diffusion conditions has been illustrated in the literature ${ }^{122}$ for ortho to para hydrogen conversion reaction.

\section{Nomenclature}

$a=$ pore radius, $\quad \bar{a}=$ average pore radius $\quad[\mathrm{cm}]$

$A_{S}=$ cross sectional area of pellet $\cdot\left[\mathrm{cm}^{2}\right]$

$C_{i 0}=$ concentration of gas $i$ at pore entrance $\left[\mathrm{g}-\mathrm{mole} / \mathrm{cm}^{3}\right]$

$C_{i}(a)=$ flow constant of gas $i \quad\left[\mathrm{~cm}^{2} / \mathrm{sec}\right]$

$D_{e i}=$ effective diffusivity of gas $i \quad\left[\mathrm{~cm}^{2} / \mathrm{sec}\right]$

$D_{i}(a)=$ diffusivity of gas $i$ in capillary tube $\quad\left[\mathrm{cm}^{2} / \mathrm{sec}\right]$

$D_{i-j}=$ molecular diffusivity between gas $i$ and $j\left[\mathrm{~cm}^{2} / \mathrm{sec}\right]$

$E_{f}=$ effectiveness factor $[-]$

$f \quad=$ effective volume void fraction

$k=$ tortuosity factor $[-]$

$\begin{array}{llr}k_{S} & =\text { reaction rate constant } & {[\mathrm{cm} / \mathrm{sec}]} \\ L & =\text { pellet height } & {[\mathrm{cm}]}\end{array}$

$\begin{array}{llr}L & =\text { pellet height } & {[\mathrm{cm}]} \\ M & =\text { molecular weight } & {[\mathrm{g} / \mathrm{g}-\mathrm{mole}]}\end{array}$

$N=$ flux $\quad\left[\mathrm{g}-\mathrm{mole} / \mathrm{cm}^{2} \cdot \mathrm{sec}\right]$

$P=$ pressure, $\quad P_{0}=$ atmospheric pressure [atm]

$Q=$ permeability flow rate $\quad\left[\mathrm{g}-\mathrm{mole} / \mathrm{cm}^{2} \cdot \mathrm{sec}\right]$

$r(a)=$ reaction rate $\quad[\mathrm{g}-\mathrm{mol} / \mathrm{sec}]$

$R_{g}=$ gas constant $\quad\left[\mathrm{cm}^{3} \cdot \mathrm{atm} / \mathrm{g} \cdot \mathrm{mole} \cdot{ }^{\circ} \mathrm{K}\right]$

$S=$ surface area, $\quad S_{t}=$ total surface area calculated by $S_{t}=2 \int_{0}^{V t} d V / a, S_{B E T}=B E T$ area $\quad\left[\mathrm{cm}^{2} / \mathrm{g}\right]$

$=$ temperature

$=$ mean molecular velocity

= pore volume, $\quad V_{\mathrm{c}}=$ total pore volume

$y=$ mole fraction $\quad V_{t}=$ total pore volume $\left[\mathrm{cm}^{3} / \mathrm{g}\right]$

Greek letters

$\varepsilon \quad=$ porosity $[-]$ 
$\lambda=$ mean free path,

$\lambda_{0}=-$ at atmospheric pressure

$\mu \quad=$ gas viscosity

$\rho_{P} \quad=$ pellet density

Superscripts

$D=$ non-reactive diffusion, $P=$ permeability,

$R=$ reaction

Subscript

$i=\operatorname{gas} i$

\section{Literature Cited}

1) Evans, R. B., G. M. Watson, and E. A. Mason: J.Chem. Phys., 35, 2076 (1961)

2) Henry, J. P., Chennakesavan, and J. M. Smith: A. I.Ch. E. $J ., 7,10(1961)$

\section{[cm] \\ $[\mathrm{g} / \mathrm{cm} \cdot \mathrm{sec}]$ \\ $\left[\mathrm{g} / \mathrm{cm}^{3}\right]$}

\title{
ON MOLECULAR DISTILLATION, VAPOR-LIQUID RELATIONSHIPS FOR E.H. P.-mT. C. P. SYSTEM
}

\author{
HAJIMU UYEHA AND YUTAKA HAGIHARA** \\ Dept. of Chem. Eng., Doshisha University, Kyoto, Japan
}

\begin{abstract}
Measurements of the vapor-liquid relationship for E. H. P.-mT. C. P. system were performed by use of an agitator vessel under a residual air pressure less than $1.5 \times 10^{-3} \mathrm{mmHg}$. The results are as follows: (1) On molecular distillation, there are vapor-liquid relationships for each operation liquid surface temperature $\left(T_{s}\right)$. (2) At low liquid surface temperature, E. H. P.-mT. C. P. system behaves as an ideal solution, but deviates at high liquid surface temperature. (3) In composition range $20 \mathrm{~mol} \%$ to $80 \mathrm{~mol} \%$, the change in the ratio of $\boldsymbol{\alpha}_{\mathrm{ob}} / \boldsymbol{\alpha}_{\mathrm{th}}$ to $x$ is approximately shown as a quadratic equation : $\alpha_{\mathrm{ob}} / \alpha_{\mathrm{ih}}=b-a(x-0.5)^{2}$. $a$ is the constant value for the same system. $b$ is shown as an equation of the first degree of $T_{s}$. The empirical formula derived in this work, shows agreement with the observed values in compcsition range 20 mole $\%$ to 80 mole $\%$.
\end{abstract}

\section{Introduction}

In molecular distillation, the evaporated molecules move directly to the condenser without collision with other molecules. When a sufficiently low temperature is employed in the condenser, re-evaporation from the condenser can be neglected. Consequently the evaporated molecules do not return to the distilland. In molecular distillation, therefore, there is a completely nonequilibrant condition ${ }^{4)}$. The concept of equilibrium distillation cannot be applied to molecular distillation. In molecular distillation, rather new knowledge of the vapor-liquid relationship is required.

An experiment in distillation of a mixture of di-2ethyl-hexyl-phthalate (E. H. P.) and $m$-tricresyl-phosphate ( $\mathrm{mT}$. C. P.) was ferformed by use of an agitator vessel under a residual air pressure less than $1.5 \times 10^{-3}$ $\mathrm{mmHg}$. In this range of residual air pressure, there are no effects of residual air pressure on the evaporation rate $^{5)}$. The vapor-liquid relationships were measured and deviations from ideal behavior were shown as a

* Received on January 20, 1969

** Chem. Lab., Defense Academy, Yokosuka, Japan function of the ratio $\alpha_{\circ b}$ to $\alpha_{\mathrm{th}}$. The experimental results and discussion follow.

\section{Experiments}

The experimental apparatus is shown in Fig. 1. A flask is immersed in heating medium (SK oil No. 240). In molecular distillation, evaporation occurs only on the liquid surface. To evaporate from the uniform liquid surface, the liquid phase is agitated by a magnetic stirrer (1). The vapor is condensed by the condenser (2). The condensed vapor runs down through the flow-meter (3) and returns to the pot still (4). In the steady state, the condensed vapor is run down to the sampling box (5), and then the residual air pressure in the pot still is increased to atmospheric pressure. The liquid in the pot still is sampled by squirt. The condensed vapor and the liquid in the pot still are used to determine mole per cent composition through use of an ordinary Abbe refractometer to an accuracy of $0.5 \%$. In Fig. 2 , the refractive index is plotted against mole per cent concentration of E.H.P. The temperature of the liquid surface is measured with a pair of thermocouples ${ }^{6)}$ (6). 